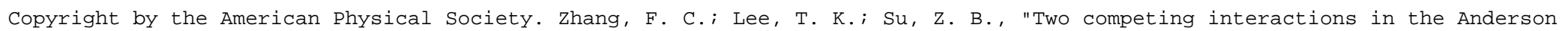
lattice model," Phys. Rev. B 35, 4728 DOI: http://dx.doi.org/10.1103/PhysRevB.35.4728

\title{
Two competing interactions in the Anderson lattice model
}

\author{
F. C. Zhang \\ Center for Theoretical Physics, Department of Physics and Astronomy, University of Maryland, College Park, Maryland 20742 \\ and Theoretische Physik, Eidgenössische Technische Hochschule Zürich-Hönggerberg, CH-8093 Zurich, Switzerland \\ T. K. Lee \\ Department of Physics, Virginia Polytechnic Institute and State University, Blacksburg, Virginia 24061 \\ Z. B. Su \\ Department of Physics, City College of the City University of New York, New York, New York 10031 \\ (Received 21 July 1986; revised manuscript received 7 November 1986)
}

\begin{abstract}
The vertex function of the $\mathrm{SU}(N)$ Anderson lattice model is calculated by treating the intersite coupling perturbatively. There are two different scattering processes that contribute to the effective interactions. In one process the low-frequency Kondo resonance dominates and the effective interaction between quasiparticles is favorable for $p$-wave Cooper pairing at small values of $k_{F} R$. In the other process all frequencies contribute and the effective interaction is against $p$-wave pairing for small $k_{F} R$. This latter interaction is antiferromagnetic in nature.
\end{abstract}

\section{INTRODUCTION}

Recently heavy-fermion systems have attracted many physicists because of their exotic properties. ${ }^{1,2}$ One of the most important and interesting puzzles is the mechanism causing the attractive interaction and hence the symmetry property of Cooper pairs.

The similarity between various properties of heavyfermion superconductors and ${ }^{3} \mathrm{He}$ has produced a number of conjectures ${ }^{3,4}$ that a nonconventional attractive interaction may be responsible for the Cooper pairing. On the other hand, Razafimandimby et al. ${ }^{5}$ have proposed the conventional electron-phonon mechanism for the heavyfermion superconductor $\mathrm{CeCu}_{2} \mathrm{Si}_{2}$.

During the last year several groups ${ }^{6-8}$ have started to calculate the effective interaction between electrons based on the $\mathrm{SU}(N)$ Anderson lattice model. In this model both the conduction electron and $f$ electron are assumed to have the same spin degeneracy $N$. So far two different approaches have been adopted. In Refs. 7 and 8 the Kondo-boson approach is used while the GoldstoneFeynman diagrammatic method $^{9}$ is used in Ref. 6.

With the use of the result of the mean-field treatment in the Kondo-boson approach, the effective interaction or the vertex function between quasiparticles is calculated. It is found that the coupling constants are repulsive for $s$-wave and $p$-wave pairings but attractive for $d$-wave pairing. ${ }^{7,8}$ Several other groups ${ }^{10}$ have proposed $d$-wave pairing as a possible candidate for heavy-fermion superconductivity. An argument against $d$-wave pairing is put forward by two of the present authors (F.C.Z. and T.K.L.) in a recent paper. ${ }^{11}$

In this paper we shall use the diagrammatic approach ${ }^{9}$ to calculate the effective interaction between conduction electrons in the $\mathrm{SU}(N)$ Anderson lattice model. The result of the single-Kondo-impurity system is used as a basis to treat the intersite coupling due to the virtual ex- change of conduction electrons in the perturbative series. A preliminary derivation was given in Ref. 6 . Besides the well-know repulsion between electrons in different spin states scattered from a single magnetic ion, we show that there are two different contributions to the effective interaction coupling. One of the interactions favors $p$-wave pairing of electrons with parallel spins, while the other one is against parallel-spin correlation between electrons on two sites.

The interaction that favors $p$-wave pairing at values of $k_{F} R<2$, is an oscillatory function of $k_{F} R$, where $k_{F}$ is the Fermi momentum and $R$ is the separation between two magnetic ions. Depending on the explicit lattice structure, it is possible to have an attractive $p$-wave coupling constant. When this interaction, obtained via virtual exchange of low-frequency quasiparticles, is evaluated in wave-vector space using the jellium approximation, it agrees with the Kondo-boson approach ${ }^{7,8}$ where the $p$ wave pairing is found to be unfavorable and independent of the underlying lattice structure. The discrepancy between the results using and not using the jellium approximation indicates that the assumption of a uniform background in the jellium approximation is inappropriate for the Kondo lattice. We expect this because the quasiparticles have strong $f$-electron character, and $f$ electrons are localized at the magnetic ion site. We find that the breakdown of Galilean invariance is one of the important differences between heavy-fermion superconductors and other superconductors.

The interaction that impedes parallel-spin correlation is obtained by virtual exchange of electrons at all frequencies, not just at low frequencies as is usually considered. This high-frequency contribution has been completely neglected in earlier works. ${ }^{6-8}$ We find that this interaction may be responsible for antiferromagnetic correlation in heavy-fermion systems.

The paper is organized as follows. In Sec. II, the per- 
turbative methods and results for the single Kondo impurity are briefly reviewed. The Green's functions for quasiparticles are introduced. The on-site vertex function is derived in Sec. III. In Sec. III A, the low-frequency contribution to the vertex function is calculated in both wave-vector space (using the jellium approximation) and the real space. The contribution of this interaction to $p$ wave pairing is discussed. In Sec. III B, the highfrequency contribution is shown to produce a repulsive interaction for $p$-wave pairing. In real space this effective interaction is shown to be antiferromagnetic in nature. Finally in Sec. IV, we summarize the results and examine the approximations used.

\section{REVIEW OF THE PERTURBATION METHODS AND ITS RESULTS}

The dense Kondo system is best described by the Anderson lattice model. The Hamiltonian is given by

$$
\begin{aligned}
H= & \sum_{\mathbf{k}, m} \varepsilon_{\mathbf{k}} C_{\mathbf{k} m}^{\dagger} C_{\mathbf{k} m}+\varepsilon_{f} \sum_{m} X_{m j}^{\dagger} X_{m j} \\
& +\sum_{\mathbf{k}, m, j}\left(V_{\mathbf{k}_{m}}^{j} C_{\mathbf{k} m}^{\dagger} X_{m j}+\text { H.c. }\right),
\end{aligned}
$$

where the projection operator on site $j, X_{m j}$, changes the localized $f$ configuration from $\left|f^{1}, m\right\rangle$ to $\left|f^{0}\right\rangle$. There is a total of $N=2 J+1$ degenerate $\left|f^{1}, m\right\rangle$ states (the angular momentum $m$ runs from $-J$ to $J$ ). In the Kondo limit, the bare $f$-level energy $\varepsilon_{f}<0$, and the on-site Coulomb repulsion has been set infinite. The hybridization matrix

$$
V_{\mathbf{k} m}^{j}=\frac{1}{\sqrt{N_{s}}} V_{\mathbf{k} m} \exp \left(i \mathbf{k} \cdot \mathbf{R}_{j}\right)
$$

with $N_{s}$ the number of lattice sites. For simplicity the crystal symmetry shall be neglected here. Equation (2.1) is the popular $\mathrm{SU}(N)$ Anderson lattice model, ${ }^{12}$ where the conduction electrons have $N$ degenerate spin states as do the $f$ electrons. Each band is assumed to have a constant density of states $\rho_{0}=n_{e} / N D$ where $D$ is the half bandwidth and $n_{e}$ is the density of conduction electrons.

The Anderson lattice model has been recently studied by Brandow ${ }^{13}$ using a variational wave function, and by Rice and Ueda ${ }^{14}$ using the Gutzwiller variational approximation. Various groups have studied the model by using mean-field theory in the Kondo-boson method. $7,8,12,15,16$ Here we take a different approach. We assume that the system is basically a collection of uncorrelated Kondo impurities above the Kondo temperature. As the temperature decreases, the intersite coupling turns on. Thus we shall use the result of a single-Kondo-impurity model as a basis to understand the lattice model and to treat the intersite coupling perturbatively.

We shall briefly review the results for the singleimpurity model. Details are to be found in Ref. 17. The perturbative technique used to study the Anderson lattice model was previously developed by Grewe and Keiter ${ }^{9}$ and involves combination of Goldstone and Feynman diagrams. The Goldstone diagrammatic rule is used for processes occurred on a single site. The Feynman rule is used to connect diagrams on different sites. Self-energies that only involve a single site have been extensively stud- ied in the last couple of years. ${ }^{17-23}$ Very accurate results have been obtained for the single-impurity problem. Using Grewe and Keiter's approach, we can calculate the vertex functions in series of intersite coupling. Based on the $1 / N$ expansion idea, we shall only include the lowestorder single-site self-energy for the $f$ hole (singlet state). This self-energy is of the form

$$
S_{0}(z)=\sum_{\mathbf{k}, m} \frac{\left|V_{\mathbf{k} m}\right|^{2} f_{k}}{z+\varepsilon_{\mathbf{k}}-\varepsilon_{f}},
$$

where $f_{k}$ is the Fermi distribution function. The reason for using only the lowest-order self-energies in this paper is that analytical results can be obtained easily. These results will have the same qualitative behavior as found from using higher-order corrections. One of the authors (T.K.L.) has calculated the vertex functions ${ }^{6}$ using the approximate spectral density obtained from the noncrossing approximation, ${ }^{17}$ and the result is in good agreement with the results given below.

In the Kondo regime and at zero temperature, the function $\left[z-S_{0}(z)\right]^{-1}$, which will appear quite often, can be approximately written in the form ${ }^{24}$

$$
\frac{1}{z-S_{0}(z)} \cong \frac{a_{0}}{z-E_{0}}+\frac{1-a_{0}}{z}
$$

with $a_{0}=T_{0} / N \Delta<<1$, and

$$
\Delta=\frac{1}{N_{s}} \sum_{\mathbf{k}}\left|V_{\mathbf{k} m}\right|^{2} \delta\left(\omega-\varepsilon_{\mathbf{k}}\right)=\rho_{0} V^{2} .
$$

In Eq. (2.3), the lifetime broadening has been neglected for simplicity. $E_{0}$ is the energy of the Kondo singlet state, and is given by the equation:

$$
T_{0}=\varepsilon_{f}-E_{0}=D \exp \left(\varepsilon_{f} / N \Delta\right),
$$

where $D$ is the half bandwidth, and $T_{0}$ is the familiar definition of the Kondo temperature.

The spectral density obtained ${ }^{17}$ for a single impurity consists of a low-frequency "spin" excitation peak and a high-frequency "charge" excitation peak. The first and second terms of Eq. (2.3) are related to the low- and high-frequency excitations respectively. Hence different contributions to the vertex function are expected from these two terms.

So far we have only discussed the result for the singleimpurity model. For the lattice, as temperature falls below the Kondo temperature $T_{0}$, the conduction electrons interact with local $f$ electrons coherently. A quasiparticle band with a very heavy effective mass is formed. This coherent quasiparticle band has been obtained by several workers ${ }^{12-15,25-27}$ using different approaches.

In Refs. 26 and 27, the coherent quasiparticle bands for the periodic Anderson model in the Kondo regime were derived. Although the result presented there uses more realistic hybridization matrix elements, it is easy to generalize that result to the present $\mathrm{SU}(N)$ model of Eq. (2.1). The Green's function for the renormalized conduction electrons is of the form

$$
\widetilde{g}_{c}(\mathbf{k}, i \omega)=\frac{i \omega-T_{0}}{\left(i \omega-E_{\mathbf{k}}^{+}\right)\left(i \omega-E_{\overline{\mathbf{k}}}^{-}\right)},
$$


where

$$
E_{\mathrm{k}}^{ \pm}=\frac{1}{2}\left(\varepsilon_{\mathrm{k}}+T_{0}\right) \pm \frac{1}{2}\left[\left(\varepsilon_{\mathrm{k}}-T_{0}\right)^{2}+4 V^{2} T_{0} / N \Delta\right]^{1 / 2} \text {. }
$$

The $+(-)$ label refers to the upper (lower) hybridized band. Identical results are obtained in the mean-field treatment ${ }^{7,8,12}$ of the Kondo-boson approach. Assuming the Fermi level, where we have $E^{-}\left(k_{F}\right)=0$ and $\varepsilon\left(k_{F}\right)=1 / N \rho_{0}$, is near the top of the lower bands, one finds the mass enhancement to be

$M^{*} / M=\left(\frac{\partial E-(\varepsilon)}{\left.\partial \varepsilon\right|_{k_{F}}}\right)^{-1}=\rho^{*} / \rho_{0}=\frac{1}{N \rho_{0} T_{0}} \gg 1$

Here $\rho^{*}$ is the quasiparticle density of states per spin. The Fermi velocity $\widetilde{v}_{F}=v_{F}\left(M / M^{*}\right)$ is greatly reduced. Thus these quasiparticles near the Fermi level are essentially $f$-like. The wave function renormalization for the conduction electrons at the Fermi level is given by $\left(M / M^{*}\right)^{1 / 2}$.

\section{THE VERTEX FUNCTION AND THE $\boldsymbol{p}$-WAVE COUPLING CONSTANT}

The Fourier transform of the conduction-electron vertex function, defined by

$$
\left\langle-T_{\tau} C_{\mathbf{k}_{1} m}^{\dagger}\left(\tau_{1}\right) C_{\mathbf{k}_{2} m}^{\dagger}\left(\tau_{2}\right) C_{\mathbf{k}_{3} m}\left(\tau_{3}\right) C_{\mathbf{k}_{4} m}\left(\tau_{4}\right)\right\rangle,
$$

is evaluated in this section. Its zeroth order (in the spirit of the $1 / N$ expansion) is the on-site vertex, and is plotted diagrammatically in Fig. 1 with momenta and energies $P_{l}=\left(\mathbf{k}_{l}, i \omega_{l}\right), l=1,2,3$, and 4. Notice that in Fig. 1(a) the wiggly, dashed, and solid lines represent the configurations $\left|f^{1}, m\right\rangle,\left|f^{0}\right\rangle$, and the conduction electrons, respectively. Using the contour integral representation, ${ }^{28}$ the on-site vertex $\Gamma_{m m^{\prime}}^{\mathrm{I}}$ can be easily written down:

$$
\Gamma_{m m^{\prime}}^{\mathrm{I}}\left(p_{1}, p_{2} ; p_{3}, p_{4}\right)=\sum_{j}\left[\Gamma_{j}^{\mathrm{I}}\left(p_{1}, p_{2} ; p_{3}, p_{4}\right)-\delta_{m m^{\prime}} \Gamma_{j}^{\mathrm{I}}\left(p_{1}, p_{2} ; p_{4}, p_{3}\right)\right]
$$

in which

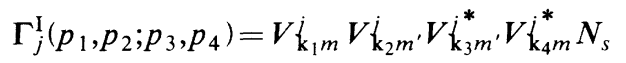

$$
\begin{aligned}
& \times \frac{1}{Z_{0}} \oint \frac{d z e^{-\beta z}}{2 \pi i} \frac{1}{\left[z-S_{0}(z)\right]\left(z+i \omega_{1}-\varepsilon_{f}\right)\left[z+i \omega_{1}-i \omega_{4}-S_{0}\left(z+i \omega_{1}-i \omega_{4}\right)\right]\left(z+i \omega_{3}-\varepsilon_{f}\right)}
\end{aligned}
$$

and $Z_{0}$ is the partition function of one single site. In the present approximation, $Z_{0}=\exp \left(-\beta E_{0}\right)$.

At zero temperature, the contour integral in Eq. (3.2) can be carried out by taking into account the pole contributions only. After the contour integration is done, we make the analytic continuation: $i \omega_{l} \rightarrow \omega_{l}+i \delta$ and let $\delta \rightarrow 0$ in the end. The momentum and energy transfers are $\mathbf{q}=\mathbf{k}_{4}-\mathbf{k}_{1}$ and $v=\omega_{4}-\omega_{1}$, respectively. By making

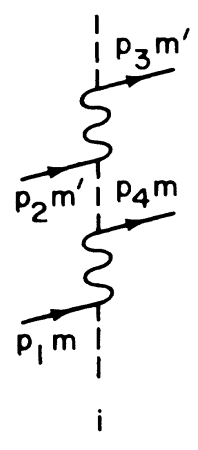

(a)

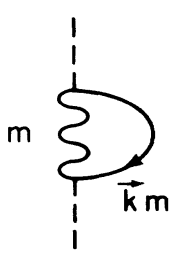

(b)
FIG. 1. (a) The Goldstone diagrammatic representation for the on-site (at site $i$ ) Landau vertex function. The wiggly, dashed, and solid lines represent the configuration $\left|f^{1}, m\right\rangle$, $\left|f^{0}\right\rangle$, and the conduction electron, respectively. Each dashed line includes the lowest order self-energy (in $1 / N$ expansion), whose irreducible diagram is shown in (b). $v \rightarrow 0$, then $\omega_{1} \rightarrow \varepsilon_{\mathbf{k}_{1}}$ and $\omega_{2} \rightarrow \varepsilon_{\mathbf{k}_{2}}$, we obtain the on-site vertex at the Fermi surface

$$
\begin{aligned}
& \Gamma_{m m^{\prime}}^{\mathrm{I}}\left(\mathbf{k}_{1}, \mathbf{k}_{2} ; \mathbf{q}\right) \\
& \quad=\left(1-\delta_{m m^{\prime}}\right) V_{\mathbf{k}_{1} m} V_{\mathbf{k}_{2} m^{\prime}} V_{\mathbf{k}_{3} m^{\prime}} V_{\mathbf{k}_{4} m} \frac{\left(1+N \Delta / \varepsilon_{f}\right)}{T_{0}(N \Delta)^{2}} .
\end{aligned}
$$

This result is in agreement with Noziere's result ${ }^{29}$ obtained from the Fermi-liquid description of the Kondo problem. It also agrees with the result in Ref. 6, where the approximate form of higher-order self-energies were used in the calculation.

Equation (3.3) shows that the electrons with different spins are repulsive and the interaction between two electrons with spins parallel is zero to this order. The strong repulsive interaction between different spins makes $s$-wave pairing very unlikely in this model.

Taking the hybridization as constant, $|V|^{2} \rightarrow \Delta / \rho_{0}$, we obtain

$$
\Gamma_{m m^{\prime}}^{\mathrm{I}}=\left(1-\delta_{m m^{\prime}}\right) \frac{1}{T_{0} \rho_{0}^{2} N^{2}},
$$

where we have dropped the small correction term $N \Delta /\left|\varepsilon_{f}\right|$, since it is much smaller than 1 in the Kondo limit. Notice that $\Gamma^{I}$ is the vertex function for the conduction electrons. Since the vertex function is evaluated at the Fermi surface, we can use the quasiparticle picture discussed at the end of Sec. II. In the quasiparticle picture the vertex function is renormalized, and becomes 


$$
\widetilde{\Gamma}_{m m^{\prime}}^{\mathrm{I}}=\left(\frac{M}{M^{*}}\right)^{2} \Gamma_{m m^{\prime}}^{\mathrm{I}}=\left(1-\delta_{m m^{\prime}}\right) T_{0} .
$$

This result agrees with leading term in the Kondo-boson approach. ${ }^{7,8}$ The next leading term in the Kondo-boson approach is shown to equal to the low-frequency vertex function evaluated below.

The nonvanishing lowest-order vertex function for electrons with parallel spins involves the intersite coupling. The diagrammatic representation of which is shown in Fig. 2. The three diagrams in Fig. 2 have different outgoing external lines, and describe different scattering processes. Diagram $L$ in Fig. 2 represents the fluctuations in which the low frequency, namely, the anomalous Kondo resonance or Abrikosov-Suhl resonance ${ }^{30}$ portion of the $f$ Green's function is dominant. More specifically, it is the first term on the right-hand side (rhs) of Eq. (2.3) that contributes most. Diagram $H$ represents the fluctuations in which the higher-frequency process [or the second term on the rhs of Eq. (2.3)] is dominant. In fact this diagram represents the familiar Ruderman-Kittel-Kasuya-Yosida (RKKY) interaction. Diagram $C$ gives correction to the low-frequency fluctuation. It is of a factor $1 / N$ less than the results given by diagram $L$, and will be neglected in the rest of the paper. The lowest-order intersite vertex function can thus be written as

$$
\Gamma_{m m}^{\mathrm{II}}\left(p_{1}, p_{2} ; p_{3}, p_{4}\right)=\Gamma_{L}+\Gamma_{H},
$$

where the subindices $L$ and $H$ indicate the low- and highfrequency contribution from the spectral density of the $f$ electron. We shall consider these two terms separately.

\section{A. Low-frequency contribution}

Using $\Gamma_{j}^{\mathrm{I}}$ of Eq. (3.2), the vertex function of diagram $L$ in Fig. 2 is

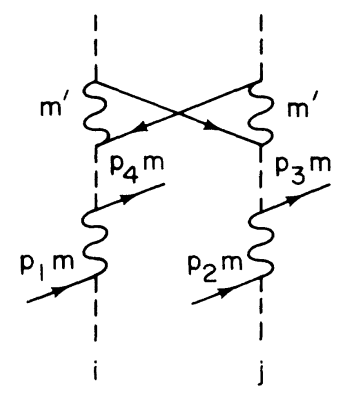

(L)

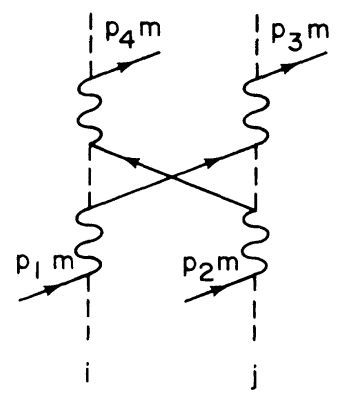

(H)

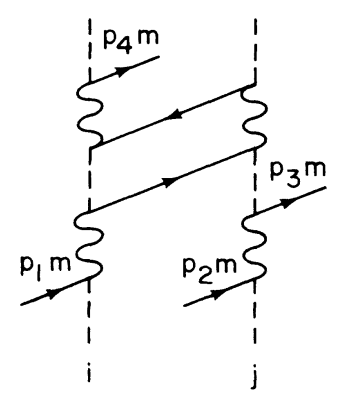

(c)

FIG. 2. Diagrammatic representation for the vertex function with the lowest-order intersite coupling (between site $i$ and $j$ ). The wiggly line ( $f$ electron) and the solid lines (conduction electrons) connected with it have the same spin index. The three diagrams represent different scattering processes as explained in the text.

$$
\begin{array}{r}
\Gamma_{L}\left(p_{1}, p_{2} ; p_{3}, p_{4}\right)=N_{s} N T \sum_{\omega_{5}, \omega_{6} \mathbf{k}_{5}, \mathbf{k}_{6}} \sum_{\substack{i, j \\
i \neq j)}} \Gamma_{i}^{\mathrm{I}}\left(p_{1}, p_{5} ; p_{6}, p_{4}\right) \Gamma_{j}^{\mathrm{I}}\left(p_{2}, p_{6} ; p_{5}, p_{3}\right) \widetilde{g}_{c}\left(i \omega_{5}, \mathbf{k}_{5}\right) \widetilde{g}_{c}\left(i \omega_{6}, \mathbf{k}_{6}\right) \\
\times \delta\left(\omega_{2}+\omega_{6}, \omega_{3}+\omega_{4}\right) \delta\left(\omega_{1}+\omega_{5}, \omega_{6}+\omega_{4}\right)-\left(p_{3} \leftrightarrow p_{4}\right) .
\end{array}
$$

The second term on the rhs of Eq. (3.7) is simply the first term by interchanging $p_{3} \leftrightarrow p_{4}$, and appears only for $m=m^{\prime} . \quad \widetilde{g}_{c}(i \omega, \mathbf{k})$ in Eq. (3.7) is the renormalized conduction-electron Green's function given by Eq. (2.5).

By making the same analytic continuation discussed for the on-site vertex function we obtain $\Gamma_{L}\left(\mathbf{k}_{1}, \mathbf{k}_{2} ; \mathbf{q}\right)$ in the static limit,

$$
\Gamma_{L}\left(\mathbf{k}_{1}, \mathbf{k}_{2} ; \mathbf{q}\right)=\Gamma^{\mathrm{I}} T_{0}\left[\chi_{L}\left(\mathbf{k}_{1}-\mathbf{k}_{2}+\mathbf{q}\right)-\chi_{L}(\mathbf{q})\right],
$$

where $\Gamma^{I}$ is given by Eq. (3.4). The static susceptibility $\chi_{L}(\mathbf{q})$ defined as the bubble diagram using Fig. $2(L)$ as vertex, is

$$
\begin{aligned}
\chi_{L}(\mathbf{q})=-N^{3} \Delta^{2} \sum_{\mathbf{R}(\neq 0)} e^{i \mathbf{q} \cdot \mathbf{R}} T \sum_{i \omega_{n}}\left[\hat{\Gamma}_{L}\left(i \omega_{n}\right)\right]^{2} \\
\times\left[\widetilde{G}_{m}\left(\mathbf{R}, i \omega_{n}\right)\right]^{2},
\end{aligned}
$$

where $\hat{\Gamma}_{L}\left(i \omega_{n}\right)$ is the contour integral in Eq. (3.2), and it is obtained by taking the limits that $i v \equiv i \omega_{4}-i \omega_{1} \rightarrow 0$ first, then $\omega_{1} \rightarrow 0$ and $i \omega_{2} \rightarrow i \omega_{n}$. One finds only the first term on the rhs of Eq. (2.3) is important, and the result is

$$
\hat{\Gamma}_{L}(i \omega)=\frac{1}{(N \Delta)^{2}} \frac{T_{0}}{\left(i \omega-T_{0}\right)^{2}} .
$$

The intersite Green's function is defined by

$$
\widetilde{G}_{m}(\mathbf{R}, i \omega)=\frac{1}{N_{s}} \sum_{\mathbf{k}}\left|V_{\mathbf{k} m}\right|^{2} e^{i \mathbf{k} \cdot \mathbf{R}_{\widetilde{g}_{c}}(\mathbf{k}, i \omega) .}
$$

Equation (3.8) is of similar form to the result for the paramagnon model ${ }^{31}$ in ${ }^{3} \mathrm{He}$ if we identify $T_{0} \chi(q)$ as the product of the paramagnon interaction constant and the Lindhard function. 
The susceptibility $\chi_{L}(q)$ may be evaluated in two ways, either by examining it in the real space numerically, or by treating the system as a jellium to evaluate $\chi_{L}(\mathbf{q})$ analytically. We shall consider the latter case first.

Under the jellium approximation, the summation over $\mathbf{R}$ and $i \omega_{n}$ in Eq. (3.9) can be carried out. We obtain

$$
\chi_{l}(q)=\frac{\alpha}{T_{0}}\left[\frac{1}{12} f\left(q / 2 k_{F}\right)+\frac{1}{N}\right],
$$

where the function $f(x)$ is defined by

$$
f(x)=\frac{1}{x} \ln \left|\frac{1+x}{1-x}\right|
$$

and

$$
\alpha=\left(N \rho_{0} k_{F}^{2} / 2 m\right)^{-1} .
$$

We have used $\varepsilon_{\mathrm{k}}=\left(k^{2}-k_{F}^{2}\right) / 2 m+1 / N \rho_{0}$ in deriving Eq. (3.12). The constant, $\alpha / N T_{0}$, in $\chi_{L}(q)$ is obtained from $R=0$ term in Eq. (3.9) under the assumption of small $\alpha$.

Because of the factor $i \omega_{n}-T_{0}$ in the denominator of $\hat{\Gamma}_{L}\left(i \omega_{n}\right)$, only conduction electrons near the Fermi surface within an energy scale of $T_{0}$ contribute to $\chi_{L}(\mathbf{q})$. The anomalous Abrikosov-Suhl resonance is dominant, and $\chi_{L}(q)$ describes the low-frequency spin fluctuation.

The vertex function $\Gamma_{L}$ obtained from $\chi_{L}(q)$ of Eq. (3.12) agrees exactly with the result of Refs. 7 and 8, although their result is derived in a completely different approach. They have used $\chi_{L}(q)$ to calculate the Fermiliquid parameters and found out that $p$-wave coupling is repulsive while $d$-wave coupling is attractive. We shall adopt a different approach below.

We believe that $\chi_{L}(q)$ of Eq. (3.12) obtained by using the jellium approximation does not properly include the lattice effects. These lattice effects cannot be neglected in Eq. (3.9). A more accurate approach of making the frequency summation first and then examining $\chi_{L}(q)$ in real space is given below.

The intersite Green's function $\widetilde{G}_{m}(R, i \omega)$ can be evaluated analytically only if we assume the zone boundary or cutoff wave vector $k_{c} \gg 1 / R$. In this case

$$
\begin{aligned}
& \widetilde{G}_{m}(\mathbf{R}, i \omega)=-\frac{\pi \Delta}{k_{F} R} \exp \left[i k _ { F } R ( \operatorname { s g n } x ) \left[1-\alpha+\frac{\alpha}{1-i x}\right.\right. \\
& \left.\left.+i x \alpha \frac{M}{M^{*}}\right]^{1 / 2}\right]
\end{aligned}
$$

where $x=\omega / T_{0}$, and $\alpha$ is defined by Eq. (3.14).

The static susceptibility $\chi_{L}(q)$ is rewritten in the form

$$
\chi_{L}(\mathbf{q})=-\frac{1}{N T_{0}} \sum_{\mathbf{R}(\neq 0)} e^{i \mathbf{q} \cdot \mathbf{R}} V_{L}\left(k_{F} R\right)
$$

where at zero temperature

$V_{L}\left(k_{F} R\right)=\frac{\pi}{2} \int_{-\infty}^{\infty} d x \frac{1}{(1-i x)^{4}}\left[\frac{\widetilde{G}_{m}(R, i x)}{\pi \Delta}\right]^{2}$.

Substituting Eq. (3.15) into (3.17) and assuming
$M^{*} / M \gg 1$, we can easily show that, for $k_{F} R \gg 1$,

$$
V_{L}\left(k_{F} R\right) \simeq \frac{\pi}{\alpha} \frac{\cos \left(2 k_{F} R\right)}{\left(k_{F} R\right)^{3}} .
$$

Thus $V_{L}\left(k_{F} R\right)$ has the familiar form of the RKKY interaction. It is well known that the Fourier transform or the lattice sum of the RKKY interaction is an oscillating function of $k_{F} a$, where $a$ is the lattice constant. This property is completely lost in the uniform-jellium approximation given by Eq. (3.12).

The importance of background lattice structure, or the breakdown of Galilean invariance is one of the major differences between heavy-fermion systems and ${ }^{3} \mathrm{He}$ or other conventional Bardeen-Cooper-Schrieffer (BCS) superconductors. In the Anderson lattice model, the Kondo effect occurred only at the magnetic ion site. The effective potential is between electrons that are screening magnetic ions on two particular sites. We cannot treat this effect by assuming a uniform background as in the jellium approximation. While in the BCS theory, the effective interaction between electrons is through virtual exchange of phonons that are not localized. Hence the jellium approximation is fairly accurate for BCS superconductivity.

Accordingly, the effective potential in real space between quasiparticles at site separation $R$, represented by diagram $L$ in Fig. 2, is given by

$$
\begin{aligned}
\widetilde{V}_{L}(R) & =\left(M / M^{*}\right)^{2} \Gamma^{\mathrm{I}} \frac{1}{N} V_{L}\left(k_{F} R\right) \\
& =\widetilde{\Gamma}^{\mathrm{I}} \frac{1}{N} V_{L}\left(k_{F} R\right)=\frac{T_{0}}{N} V_{L}\left(k_{F} R\right) .
\end{aligned}
$$

In Fig. 3 we have plotted $V_{L}\left(k_{F} R\right)$ as a function of $k_{F} R$ for two different values of $\alpha$. Instead of using the approximate form of $\widetilde{G}_{m}$ given by Eq. (3.15) for $k_{c} R \gg 1$, we have used a finite value of $k_{c}, k_{c} / k_{F}=1.5$, to calculate the integral of Eq. (3.17) numerically. We have assumed a quadratic conduction band. The results are not very sensitive to $\alpha$, as long as $\alpha<1$. The magnitude is doubled for $k_{c} \simeq 100 k_{F}$. The oscillation of $V_{L}\left(k_{F} R\right)$ is not

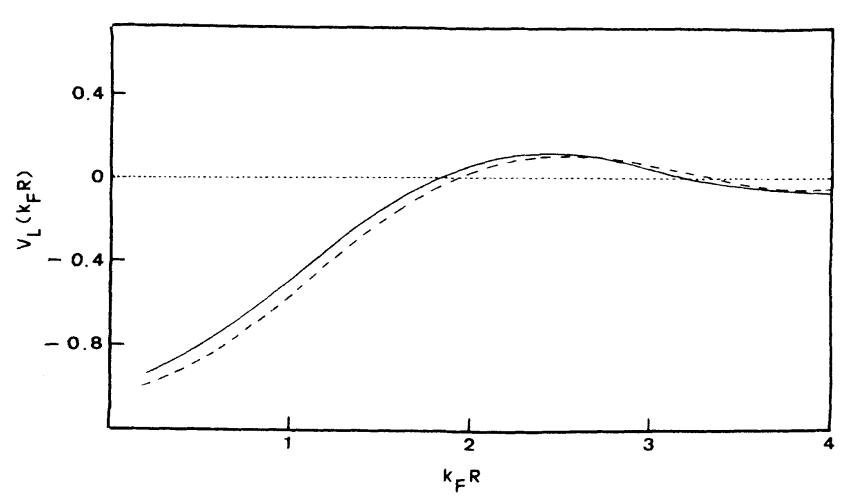

FIG. 3. The function $V_{L}\left(k_{F} R\right)$, which is proportional to the effective potential induced by the process represented by diagram $L$ in Fig. 2, is plotted as a function of $k_{F} R$ for $\alpha=\frac{1}{2}$ (dashed line) and $\alpha=\frac{1}{6}$ (solid line). The cutoff wave vector $k_{c}=1.5 k_{F}$, and $M^{*} / M=200$. 
changed qualitatively by varying $k_{c}$. The fact that $V_{L}\left(k_{F} R\right)$ is negative at small values of $k_{F} R$ surely would favor parallel spins. Electrons with unparallel spins will have to compensate for the strong hard-core repulsion given by $\Gamma^{I}$. It is intuitively natural that a repulsive core potential between unparallel spins should favor a parallel spin correlation. Hence we expect $\widetilde{V}_{L}(R)$ to provide an attractive $p$-wave coupling constant for the quasiparticles.

The superconductivity instability due to the lowfrequency excitation can be studied by summing up the corresponding ladder diagrams in diagram $L$ of Fig. 2 . The $p$-wave coupling constant obtained is of the form

$$
\tilde{\lambda}_{L}=-\frac{1}{3} \rho^{*}\left(M / M^{*}\right)^{2}\left[\Gamma_{L}(\phi)\right]_{l=1},
$$

where $M / M^{*}$ comes from the wave-function renormalization of the quasiparticles. The quantity $\left[\Gamma_{L}(\phi)\right]_{l=1}$ is given by

$$
\left[\Gamma_{L}(\phi)\right]_{l=1}=\frac{3}{2} \int_{0}^{\pi} d \phi \sin \phi \cos \phi \Gamma_{L}(\phi),
$$

where $\cos \phi=\hat{k} \cdot \hat{k}^{\prime}$ and

$$
\Gamma_{L}(\phi)=\left.\Gamma_{L}\left(\mathbf{k},-\mathbf{k} ; \mathbf{k}^{\prime},-\mathbf{k}^{\prime}\right)\right|_{k=k^{\prime}=k_{F}} .
$$

In the presence of the lattice $\Gamma_{L}\left(\mathbf{k},-\mathbf{k} ; \mathbf{k}^{\prime}-\mathbf{k}^{\prime}\right)$ is not only a function of $\mathbf{k}-\mathbf{k}^{\prime}$ but also depends on the direction of $\mathbf{k}$ with respect to the lattice vectors. To simplify matters we shall average over the direction of $\mathbf{R}$ and just find out the coupling constant as a function of the distance $R$, i.e., we define

$$
\tilde{\lambda}_{L}=\sum_{\mathbf{R}(\neq 0)} \lambda_{L}(\mathbf{R})
$$

and $\lambda_{L}(\mathbf{R})$ is evaluated by substituting Eq. (3.16) into Eqs. (3.8) and (3.20)-(3.22). Averaging $\lambda_{L}(\mathbf{R})$ over the direction of $\mathbf{R}$, we obtain

$\tilde{\lambda}_{L}(R)=\int \frac{d \Omega_{R}}{4 \pi} \lambda_{L}(\mathbf{R})=-\frac{2}{N^{2}} \frac{V_{L}(u)}{u^{2}}\left(\cos u-\frac{\sin u}{u}\right)^{2}$,

where $u=k_{F} R$. In Fig. 4, we plot $\tilde{\lambda}_{L}(R) N^{2}$ as a function

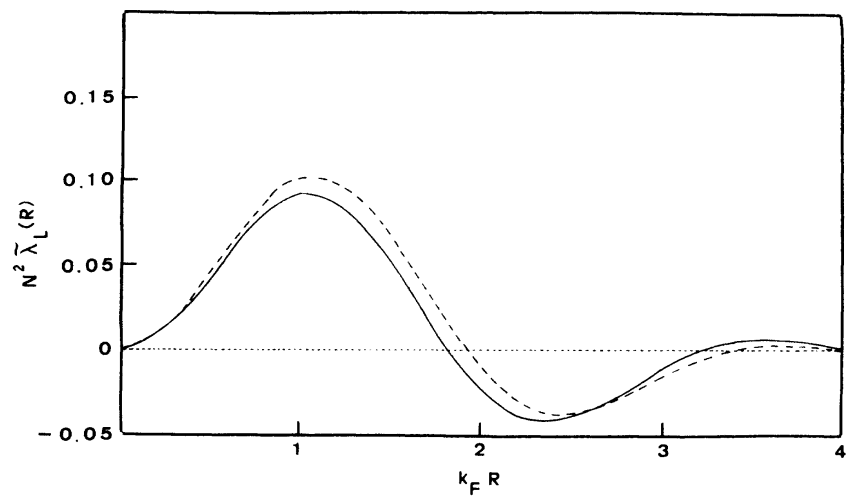

FIG. 4. $N^{2} \tilde{\lambda}_{L}(R)$, where $\tilde{\lambda}_{L}(R)$ is the contribution to the $p$ wave coupling constant represented by diagram $L$ in Fig. 2, is plotted as a function of $k_{F} R$. The parameters are the same as in Fig. 3. Positive values of $\bar{\lambda}_{L}(R)$ means favorable $p$-wave pairing. of $k_{F} R$ for $\alpha=\frac{1}{2}$ and $\alpha=\frac{1}{6}$. The positive value of $\tilde{\lambda}_{L}$ corresponds to the attractive interaction of $l=1$ component of Cooper pair. The superconducting transition temperature is $T_{c} \sim T_{0} \exp \left(-1 / \widetilde{\lambda}_{L}\right)$ for $\tilde{\lambda}_{L}>0$. We note that $\widetilde{\lambda}_{L}(R)$ is positive for $k_{F} R \leqslant 1.8$, thus it is possible to have a $p$-wave attractive interaction for magnetic ion separation $\lesssim 1.8 / k_{F}$. This is in sharp disagreement with the results obtained using the jellium approximation, ${ }^{7,8}$ Eq. (3.12), where the $p$-wave coupling is always repulsive. The reason for the inaccuracy of the jellium approximation was discussed below Eq. (3.18). We emphasize that Fig. 4 is not intended to be a quantitative proof that $p$-wave attractive interaction exists in the Kondo lattice. It mainly indicates that the possibility for $p$-wave pairing exists as contrary to the conclusion of Refs. 7 and 8 . Because of the oscillatory nature of the potential $\widetilde{V}_{L}(R)$, the lattice structure, band structure, and spin-orbit coupling are important in determining quantitatively the value of coupling constant.

\section{B. High-frequency contribution}

We now calculate the vertex function of the diagram represented by diagram $H$ in Fig. 2. Here we shall consider the case where all the spins are parallel. In analogy with $\Gamma_{L}$, we have

$\Gamma_{H}\left(\mathbf{k}_{1}, \mathbf{k}_{2} ; \mathbf{q}\right)=\Gamma^{\mathrm{I}} T_{0}\left[\chi_{H}\left(\mathbf{k}_{1}-\mathbf{k}_{2}+\mathbf{q}\right)-\chi_{H}(\mathbf{q})\right]$,

where

$$
\begin{aligned}
\chi_{H}(\mathbf{q})=-N^{2} \Delta^{2} \sum_{\mathbf{R}(\neq 0)} e^{i \mathbf{q} \cdot \mathbf{R}} T \sum_{i \omega_{n}}\left[\hat{\Gamma}_{H}\left(i \omega_{n}\right)\right]^{2} \\
\times\left[\widetilde{G}_{M}\left(R, i \omega_{n}\right)\right]^{2} .
\end{aligned}
$$

Note that the $N$ factor in $\chi_{H}$ of Eq. (3.26) is one order less than that in $\chi_{L}$ of Eq. (3.9). $\quad \Gamma_{H}\left(i \omega_{n}\right)$ is obtained from the contour integral in Eq. (3.2) by taking the limits $i v=i \omega_{3}-i \omega_{1} \rightarrow 0$, and $i \omega_{1} \rightarrow 0, i \omega_{2} \rightarrow i \omega_{n}$. Thus the second term on the rhs of Eq. (2.3) becomes important and we obtain

$$
\widehat{\Gamma}_{H}(i \omega)=\frac{1}{T_{0} N \Delta\left(\varepsilon_{f}-i \omega\right)} .
$$

A term similar to $\hat{\Gamma}_{L}(i \omega)$ but smaller by a factor of $N \Delta /\left|\varepsilon_{f}\right|$ is neglected in deriving $\hat{\Gamma}_{H}(i \omega)$.

Just as we did for $\chi_{L}(q), \chi_{H}(q)$ will be evaluated in two ways, either by using the jellium approximation or by taking the frequency sum and examining the result in real space. We shall consider the former approach first. Substitution of Eqs. (3.11) and (3.27) into (3.26) yields

$$
\chi_{H}(\mathbf{q})=-\frac{V^{4}}{T_{0}^{2}} T \sum_{i \omega} \sum_{\mathbf{k}} \frac{1}{\left(i \omega-\varepsilon_{f}\right)^{2}} \widetilde{g}_{c}(k, i \omega) \widetilde{g}_{c}(\mathbf{k}+\mathbf{q}, i \omega) \text {. }
$$

A constant, originated from $\mathbf{R}=0$ term in Eq. (3.25), has been neglected in $\chi_{H}(\mathbf{q})$. We notice that in the Kondo limit $\left|\varepsilon_{f}\right| \gtrsim D$, not only the low frequencies but all frequencies contribute to the sum in Eq. (3.26). Hence we can replace the renormalized conduction electron Green's 
function $\widetilde{g}_{c}$ by the unrenormalized form $\left(i \omega-\varepsilon_{k}\right)^{-1}$ as they only differ within the frequency range $V\left(T_{0} / N \Delta\right)^{1 / 2}$. Using this approximation we obtain

$$
\chi_{H}(\mathbf{q}) \cong \frac{1}{\left(\rho_{0} T_{0}\right)^{2}}\left(\frac{\Delta}{\varepsilon_{f}}\right)^{2} \chi_{0}(\mathbf{q}),
$$

where $\chi_{0}(\mathbf{q})$ is the familiar Lindhard function given by

$$
\chi_{0}(\mathbf{q})=\sum_{\mathbf{k}} \frac{f_{\mathrm{k}+\mathbf{q}}-f_{\mathrm{k}}}{\varepsilon_{\mathrm{k}}-\varepsilon_{\mathrm{k}+\mathbf{q}}} .
$$

Comparing $\chi_{H}(\mathbf{q})$ with $\chi_{L}(\mathbf{q})$ of Eq. (3.9), we notice that the factor $\left(i \omega-T_{0}\right)^{-4}$ in $\chi_{L}(\mathbf{q})$ makes the lowfrequency part of the spectrum to be important. As this is not the case in $\chi_{H}(\mathbf{q})$ where all frequencies contribute. Usually we would neglect $\chi_{H}(q)$ with respect to $\chi_{L}(q)$, which is due to the low-frequency Kondo peak. But the ratio

$$
\chi_{H}(q=0) / \chi_{L}(q=0) \simeq \frac{6 N\left(k_{F}^{2} / 2 M\right)}{T_{0}}\left(\frac{\Delta}{\varepsilon_{f}}\right)^{2},
$$

depending on the parameters, could be greater than one. The reason is that although the Kondo resonance peak is near the Fermi surface, it has a very small weight and only a very small portion of the spectrum $\left(\rho_{0} T_{0}<1\right)$ contributes, although the contribution of the high-frequency excitation is usually smaller by a factor of $\left(\Delta / \varepsilon_{f}\right)^{2}$, it includes all the spectrum. Thus the ratio $\left(\Delta / \varepsilon_{f}\right)^{2}\left(1 / \rho_{0} T_{0}\right)$ determines the relative importance of $\chi_{H}$ and $\chi_{L}$.

Just as $\chi_{L}(\mathbf{q})$, using the jellium approximation to calculate $\chi_{H}(q)$ is inappropriate, the lattice structure cannot be neglected. Follow the discussion given in Sec. III A, we shall rewrite $\chi_{H}(\mathbf{q})$ in the form

$$
\chi_{H}(\mathbf{q})=-\frac{1}{N T_{0}} \gamma \sum_{\mathbf{R}(\neq 0)} e^{i \mathbf{q} \cdot \mathbf{R}} V_{H}\left(k_{F} R\right),
$$

where

$$
\begin{aligned}
& \gamma=\frac{1}{\rho_{0} T_{0}}\left[\frac{\Delta}{\varepsilon_{f}}\right]^{2} \\
& V_{H}\left(k_{F} R\right)=\frac{\pi}{2} \int_{-\infty}^{\infty} \frac{d y}{\left(\frac{i y}{N \rho_{0} \varepsilon_{f}}-1\right]^{2}}\left[\frac{\widetilde{G}_{m}(R, i y)}{\pi \Delta}\right]^{2}
\end{aligned}
$$

and

$$
y=\frac{\omega}{T_{0}} \frac{M}{M^{*}}=N \rho_{0} \omega
$$

in Eq. (3.32). Substituting Eq. (3.11) into (3.32), we calculate $V_{H}\left(k_{F} R\right)$ numerically for a finite cutoff wave vector $k_{c}=1.5 k_{F}$. The results are plotted in Fig. 5 for two values of $\alpha: \quad \alpha=\frac{1}{2}$ (dashed line) and $\alpha=\frac{1}{6}$ (solid line). The other two parameters are $M^{*} / M=200$ and $N \rho_{0}\left|\varepsilon_{f}\right|=1$.

The effective potential in real space between quasiparticles separated by $R$, represented by diagram $H$ in Fig. 2, is given by

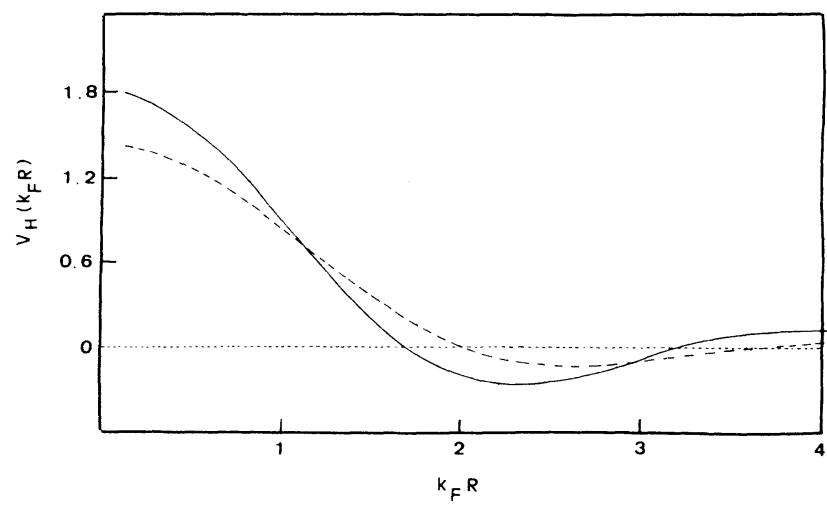

FIG. 5. The function $V_{H}\left(k_{F} R\right)$, which is proportional to the effective potential induced by the process represented by diagram $H$ in Fig. 2, is plotted as a function of $k_{F} R$. The parameters are the same as in Fig. 3. We have chosen $N \rho_{0}\left|\varepsilon_{f}\right|=1$.

$$
\widetilde{V}_{H}(R)=\left(M / M^{*}\right)^{2} \Gamma^{\mathrm{I}} \frac{1}{N} \gamma V_{H}\left(k_{F} R\right)=\frac{T_{0}}{N} \gamma V_{H}\left(k_{F} R\right),
$$

where $V_{H}\left(k_{F} R\right)$ similar to $V_{L}\left(k_{F} R\right)$ is an oscillatory function of $k_{F} R$. But contrary to $V_{L}, V_{H}\left(k_{F} R\right)$ is repulsive for $k_{F} R \lesssim 2$.

Thus this effective potential $\widetilde{V}_{H}(R)$ is against parallel spin correlation. To demonstrate it quantitatively we shall calculate the $p$-wave coupling constant contributed by $\widetilde{V}_{H}$. Following the derivation of Eqs. (3.20)-(3.23), we obtain

$$
\tilde{\lambda}_{H}=\sum_{\mathbf{R}(\neq 0)} \lambda_{H}(\mathbf{R})
$$

and the angular average of $\lambda_{H}(\mathbf{R})$ is given by

$$
\begin{aligned}
\tilde{\lambda}_{H}(R) & =\int \frac{d \Omega_{R}}{4 \pi} \lambda_{H}(\mathbf{R}) \\
& =-\frac{2}{N^{2}} \gamma \frac{V_{H}(u)}{u^{2}}\left(\cos u-\frac{\sin u}{u}\right)^{2},
\end{aligned}
$$

where $u=k_{F} R$. In Fig. $6,\left(N^{2} / \gamma\right) \widetilde{\lambda}_{H}(R)$ is plotted as a

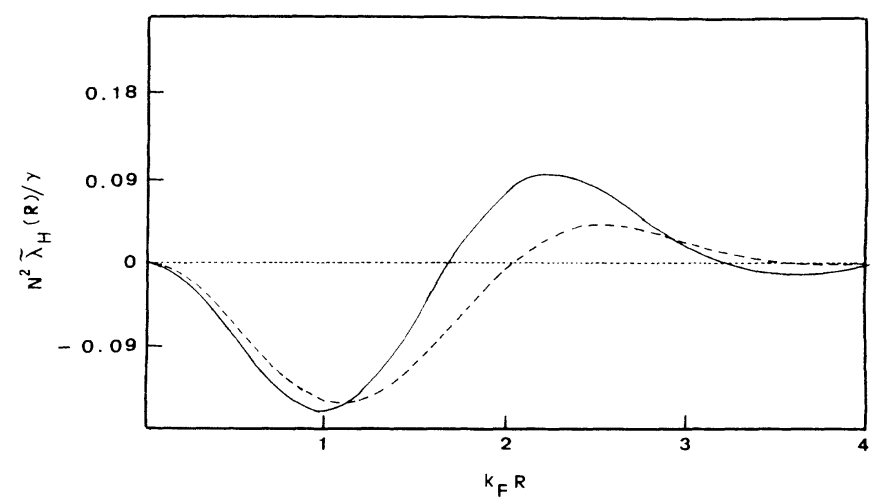

FIG. 6. $\gamma^{-1} N^{2} \widetilde{\lambda}_{H}(R)$, where $\tilde{\lambda}_{H}(R)$ is the contribution to the p-wave coupling constant calculated from diagram $H$ in Fig. 2, is plotted as a function of $k_{F} R$. The parameters are the same as in Fig. 5. 
function of $k_{F} R$ for $\alpha=\frac{1}{2}$ (dashed) and $\alpha=\frac{1}{6}$ (solid). All other parameters are the same as in Fig. 5. Comparing Figs. 4 and 6 , we see that $\widetilde{\lambda}_{L}(R)$ favors $p$-wave pairing as it is positive for $k_{F} R \lesssim 1.8$, but $\tilde{\lambda}_{H}(R)$ provides a repulsive $p$-wave coupling constant. The ratio of the magnitudes of $\left|\tilde{\lambda}_{H}(R)\right| /\left|\tilde{\lambda}_{L}(R)\right|$ is of order $\gamma$. Thus unless $\gamma$ is very small, the total $p$-wave coupling constant $\tilde{\lambda}_{=} \tilde{\lambda}_{H}+\tilde{\lambda}_{L}$ may not be large enough for Cooper pairing.

Here we notice that $\gamma$ defined by Eq. (3.31) is in fact the ratio of RKKY interaction, which is of order $\left(1 / \rho_{0}\right)\left(\Delta / \varepsilon_{f}\right)^{2}$, and the Kondo temperature $T_{0}$. It is argued by many groups ${ }^{12,14,16,32,33}$ that this ratio $\gamma$ determines whether the ground state of the Kondo lattice is magnetic or singlet. In this paper we have assumed $\gamma<1$ so that the Kondo screening effect is dominant.

But the magnetic state mentioned above is referring to the usual RKKY interaction between the magnetic ions or localized $f$ electrons without including Kondo renormalization. Here $\Gamma_{H}$ or $\widetilde{V}_{H}(R)$ is the effective interaction between quasiparticles not between localized $f$ electrons. Since the quasiparticle has $f$ character it retained part of this interaction. We should not confuse $\widetilde{V}_{H}(R)$ with the usual RKKY interaction. Incidentally, this relation between $\widetilde{V}_{H}(R)$ and the usual RKKY interaction shows a possible link between localized and itinerant magnetism.

So far we have only looked at the vertex functions [Eq. (3.6)] for parallel spins, i.e., spins in Fig. 2 are all parallel. For electrons with unparallel spins, where $m_{1}=m_{4}=m$ and $m_{2}=m_{3}=m^{\prime} \neq m$, the vertex functions for diagrams $L$ and $H$ in Fig. 2 have the form $-\Gamma^{\mathrm{I}} T_{0} \chi_{L}(\mathbf{q})$ and $\Gamma^{\mathrm{I}} T_{0} \chi_{H}\left(\mathbf{k}_{1}-\mathbf{k}_{2}+\mathbf{q}\right)$, respectively. The corresponding effective potentials between quasiparticles in real space are then given by

$$
H_{m m^{\prime}}^{(1)}=\widetilde{V}_{L}(R) a_{m}^{\dagger}(\mathbf{R}) a_{m}(\mathbf{R}) a_{m^{\prime}}^{+}(0) a_{m^{\prime}}(0)
$$

and

$$
H_{m m^{\prime}}^{(2)}=\widetilde{V}_{H}(R) a_{m}^{\dagger}(\mathbf{R}) a_{m^{\prime}}(\mathbf{R}) a_{m^{\prime}}^{\dagger}(0) a_{m}(0),
$$

where $a_{m}(R)$ is the Fourier transform of the annihilation operator $a_{k m}$ of the quasiparticle in coherent band $E_{\mathrm{k}}^{-}$of Eq. (2.6). $\widetilde{V}_{L}(R)$ and $\widetilde{V}_{H}(R)$ are defined by Eqs. (3.19) and (3.33). Due to the strong repulsion given by $\widetilde{\Gamma}^{I}$ of Eq. (3.5), $\widetilde{V}_{L}(R)$, hence $H_{m m}^{(1)}$, is unlikely to be important. But $H_{m m^{\prime}}^{(2)}$ of Eq. (3.36) represents a new kind of spin correlation between quasiparticles. As shown in Fig. 5, $\widetilde{V}_{H}(R) \propto V_{H}\left(k_{F} R\right)$ is positive for $k_{F} R \lesssim 1.5$. For the spin $\frac{1}{2}$ case, $\widetilde{V}_{H}(R)$ gives us the antiferromagnetic correlation.

As discussed below in Eq. (3.35), if $\gamma$ or $\widetilde{V}_{H}(R)$ is not very small compared to $\widetilde{V}_{L}(R)$, then $p$-wave Cooper pairing may be unlikely. But antiferromagnetic instability due to $\boldsymbol{H}_{\mathrm{mm}}^{(2)}$, may be possible. In any case, we would anticipate that $H_{m m^{\prime}}^{(2)}$ will induce antiferromagnetic fluctuations. Quantitative evidence will have to wait for the calculation of dynamic magnetic susceptibility.

\section{CONCLUSIONS}

Using our knowledge of the single Kondo impurity model, we calculate the vertex functions between conduction electrons for the $\mathrm{SU}(N)$ Anderson lattice model. The effective interactions between quasiparticles are directly proportional to these vertex functions evaluated on the Fermi surface.

Without including the intersite coupling, our result for the vertex function is in agreement with Noziere's result ${ }^{29}$ derived for the single Kondo impurity model using Fermi-liquid theory. It is also in agreement with the result of Kondo-boson approach. ${ }^{7,8}$

There are two kinds of processes that contribute to the lowest-order intersite coupling. In both processes the quasiparticles are exchanged virtually. These two different processes occur because the $f$ electron spectral density has two peaks: a low-frequency peak centered at Kondo temperature and a high-frequency peak centered at the $f$ electron energy level. Although the effective interaction is calculated between electrons on the Fermi surface, the high-frequency peak makes substantial contribution. The reason is that even though the Kondo peak is at low frequency it only involves a very small portion, $\rho_{0} T_{0}$, of the spectrum. Although the contribution of the highfrequency peak is smaller by a factor of $\left(\Delta / \varepsilon_{f}\right)^{2}$, it includes all the spectrum. We found that the ratio of this high-frequency and low-frequency processes is roughly

$$
\gamma=\left(\frac{\Delta}{\varepsilon_{f}}\right)^{2}\left(\frac{1}{\rho_{0} T_{0}}\right) .
$$

The low-frequency contribution to the vertex function $\Gamma_{L}$, evaluated in the wave-vector space by using the jellium approximation, agrees with the result of Kondo-boson approach. ${ }^{7,8}$ The $p$-wave Cooper pairing is found to be unfavorable. But we argue that neglect of the lattice structure is not appropriate for the Kondo lattice. The breakdown of Galilean invariance is one of the major differences between the Kondo lattice and ${ }^{3} \mathrm{He}$ or other BCS superconductors. We show by direct numerical calculation that the effective interation in real space is an oscillatory function of $k_{F} R$. It favors $p$-wave Cooper pairing at values of $k_{F} R \leqslant 1.8$. This oscillatory nature of the interaction, similar to the famous RKKY interaction, makes the jellium approximation of neglecting the discrete lattice sum inappropriate.

Contrary to $\Gamma_{L}$, the high-frequency contribution to the vertex function, $\Gamma_{H}$, evaluated in the real space provides a repulsive $p$-wave coupling constant at small $k_{F} R$. We also argue that this interaction, in fact, favors antiparallel spin correlation between quasiparticles. The ratio of the magnitude of $\Gamma_{H}$ and $\Gamma_{L}$ depends on $\gamma=\left(1 / \rho_{0} T_{0}\right)\left(\Delta / \varepsilon_{f}\right)^{2}$ and the detailed information of the lattice and band structure.

In summary, we found two competing effective interactions between quasiparticles. One interaction favors $p$ wave Cooper pairing while the other one against it. The latter seems to favor antiferromagnetic correlation. Qualitatively this result is certainly very appealing as it is compared with the experimental findings ${ }^{1}$ of the three possible ground state, the superconductor, the Fermi liquid and the antiferromagnetic, for the heavy fermion systems.

Here we like to emphasize that although the numerical values of the effective interactions seem to be a little small for $N=6$, we do not believe that this is a serious problem. 
In fact we advise against using the results of this paper quantitatively.

A more realistic model ${ }^{26,27}$ including the spin-orbit coupling for the hybridization matrix element is needed to obtain quantitative results. In the $\mathrm{SU}(N)$ model considered in this paper, the intersite coupling is always smaller by at least a factor of $1 / N$. In a more realistic model this is changed. Another important effect is that the anisotropic nature of the effective interaction is completely lost in the $\mathrm{SU}(N)$ model. Thus our $\Gamma_{L}$ and $\Gamma_{H}$ are both isotropic. But in a realistic model the effective interactions, $\Gamma_{L}$ and $\Gamma_{H}$, will both be anisotropic. Due to different kinds of matrix elements involved in $\Gamma_{L}$ and $\Gamma_{H}$, they will be anisotropic in different directions. The competition between them should give many interesting possibilities.

Before we conclude this paper it is important to point out our assumption that the intersite interaction is always much smaller than the Kondo temperature. As a consequence, the quasiparticle bands are derived without including the self-energy due to intersite coupling. And it is enough to calculate the effective interactions to lowest order of intersite coupling. This assumption is supported by the agreement between our approach and Kondo-boson approach, ${ }^{7,8}$ where the Gaussian fluctuation or the bubble diagrams are included to infinite order.

\section{ACKNOWLEDGMENTS}

We would like to thank Professor J. L. Birman, S. P. Bowen, and V. Korenman for many useful discussions. F.C.Z. wishes to thank Professor R. E. Prange for his constant encouragement. This work is partly supported by the National Science Foundation under Grants Nos. DMR-82-13768, DMR-83-03981, and PSC-BHE and the Faculty Research Award No. RF-6-65280.
${ }^{1}$ For a recent review in experiments, see, G. R. Stewart, Rev. Mod. Phys. 56, 755 (1984).

${ }^{2}$ For a recent review in theoretical aspects, see, P. A. Lee, T. M. Rice, J. W. Serene, L. J. Sham, and J. W. Wilkins, Comments Condens. Matt. Phys. 12, 99 (1986).

${ }^{3}$ C. M. Varma, Bull. Am. Phys. Soc. 29, 404 (1984).

${ }^{4}$ P. W. Anderson, Phys. Rev. B 30, 1549 (1984).

${ }^{5}$ H. Razafimandimby, P. Fulde, and J. Keller, Z. Phys. B 54, 111 (1984).

${ }^{6}$ T. K. Lee, J. Phys. C 18, L31 (1985).

${ }^{7}$ A. Auerback and K. Levin, Phys. Rev. Lett. 57, 877 (1986).

${ }^{8}$ M. Lavagna, A. J. Millis, and P. A. Lee, Phys. Rev. Lett. 58, 266 (1987); A. J. Millis and P. A. Lee (unpublished).

${ }^{9}$ N. Grewe and H. Keiter, Phys. Rev. B 24, 4420 (1981).

${ }^{10}$ D. J. Scalapino, E. Loh, Jr., and J. E. Hirsch, Phys. Rev. B 34, 8190 (1986); M. T. Béal-Monod, C. Bourbonnais, and V. J. Emery (unpublished); K. Miyake, S. Schmitt-Rink, and C. M. Varma, Phys. Rev. B 34, 6554 (1986); M. Cyrot (unpublished).

${ }^{11}$ F. C. Zhang and T. K. Lee (unpublished).

${ }^{12}$ N. Read, D. M. Newns, and S. Doniach, Phys. Rev. B 30, 384 (1984).

${ }^{13}$ B. H. Brandow, Phys. Rev. B 33, 215 (1986).

${ }^{14}$ T. M. Rice and K. Ueda, Phys. Rev. Lett. 55, 995 (1985).

${ }^{15}$ Z. Tesanovic and O. T. Valls, Phys. Rev. B 34, 1917 (1986).

${ }^{16} \mathrm{P}$. Coleman, Proceedings of the Eight Taniguchi Symposium on the Theory of Valence Fluctuating States, edited by $T$. Kasuya (Springer-Verlag, New York, in press).
${ }^{17}$ F. C. Zhang and T. K. Lee, Phys. Rev. B 30, 1556 (1984).

${ }^{18}$ H. Keiter and G. Czycholl, J. Magn. Magn. Mater. 31, 477 (1983).

${ }^{19}$ Y. Kuramoto, Z. Phys. B 33, 37 (1983).

${ }^{20}$ N. Read and D. Newns, J. Phys. C 16, 3273 (1983).

${ }^{21}$ N. Grewe, Z. Phys. 52, 193 (1982).

${ }^{22}$ P. Coleman, Phys. Rev. B 29, 3035 (1984).

23 J. W. Rasul and A. C. Hewson, J. Phys. C 17, 2555 (1984).

${ }^{24}$ The $f$-electron spectral density is determined by excitations between the $f$ particle at energy $\varepsilon_{f}$ and $f$ hole at energies determined by the two solutions of equation $x-\operatorname{Re} S_{0}(x-i \delta)=0$. One of the solutions is $x=E_{0}$, which produces the narrow Kondo peak, the other one is close to zero, producing a broad peak centered at about $\varepsilon_{f}$.

${ }^{25}$ N. Grewe and T. Pruschke, Z. Phys. B 60, 311 (1985).

${ }^{26}$ Z. Zou and P. W. Anderson, Phys. Rev. Lett. 57, 2073 (1986).

${ }^{27}$ T. K. Lee and F. C. Zhang, Phys. Rev. B 34, 8114 (1986).

${ }^{28}$ T. K. Lee and F. C. Zhang, J. Appl. Phys. 55, 1936 (1984).

${ }^{29}$ P. Nozieres, J. Low Temp. Phys. 17, 31 (1974).

${ }^{30}$ A. A. Abrikosov, Physics 2, 5 (1965); H. Suhl, Phys. Rev. 135, A515 (1965).

${ }^{31}$ K. Levin and O. T. Valls, Phys. Rev. B 17, 191 (1978).

${ }^{32}$ S. Doniach, Physica 91B, 231 (1977); P. Coleman, Phys. Rev. B 28, 525 (1983).

${ }^{33}$ N. B. Brandt and V. V. Moshchalkav, Adv. Phys. 33, 373 (1984). 